\title{
Design of Parking Guidance System based on Stackelberg Game*
}

\author{
Hongwei Zhu ${ }^{1,2}$, Lei Peng ${ }^{* 2}$, Huiyun $\mathrm{Li}^{2}$, Jianming $\mathrm{Liu}^{1}$, Xinling Zhou ${ }^{1,2}$ and Shi Dong ${ }^{1,2}$ \\ ${ }^{1}$ School of Computer Science and Information Security \\ ${ }^{1}$ Guilin University of Electronic Technology \\ ${ }^{2}$ Shenzhen Institutes of Advanced Integration Technology Chinese Academy of Sciences and The Chinese University of Hong \\ Kong \\ ${ }^{2}$ Shenzhen Institutes of Advanced Technology, Chinese Academy of Sciences \\ ${ }^{1}$ Guilin, China \\ ${ }^{2}$ Shenzhen, China \\ hw.zhu@siat.ac.cn, Lei.peng@siat.ac.cn,hy.li@siat.ac.cn, liujm_guet@qq.com,xl.zhou1@siat.ac.cn and shi.dong@siat.ac.cn
}

\begin{abstract}
As we know, parking guidance system (PGS) can help vehicles find appropriate parking lot more efficiently. Realtime guidance information delivers to vehicles; however, vehicles don't always follow the guidance due to various reasons in reality. This paper focuses this phenomenon and design the PGS in the view of multi-round Stackelberg game, aiming to recommend the target parking lot most possible be accepted by vehicles and help them complete parking. The static architecture of PGS is established and essential components such as front-end application and back-end web server are structured according to the needs of users. In dynamic procedure, the model of game between PGS and vehicles is also established and analyzed. Finally, the performance of PGS shows it can guide vehicles effectively, highly taking in account the efficiency and fairness of parking.
\end{abstract}

Key Index - PGS; Stackelberg game; Bipartite graph; Network maximum flow.

\section{INTRODUCTION}

With the development of modernization and internationalization in China, the number of vehicles has grown rapidly, the contradiction between the supply and the requirement of the parking spaces stands out increasingly. How to take full advantage of limited parking space and meet the demand for parking requirements, have become an urgent need to solve the problem[1], if the problem cannot been effectively resolved; It may cause a series of problems, such as energy waste and environmental pollution. At present, parking guidance system (PGS) is an effective way to solve the problem, realizes the parking guidance by pushing the guidance information to vehicles needed to park and help vehicles find appropriate parking lot efficiently. Therefore, PGS becomes the important part of intelligent transportation (ITS) and plays more and more important role in cities.

In general, vehicles are likely to park somewhere very near their destinations, unless there aren't any available parking spaces. The behaviour is reasonable based on human intuition and works well at the most of time, but will cause parking congestion at rush hours. On the contrary, PGS is employed some optimal guidance strategies to improve the utilization of the parking spaces in urban district, considering the factors meaningful to statistics rather than the benefit of individual vehicles. Hence, we can observe the phenomenon that many vehicles don't follow the guidance information, jammed at the gate of the nearest parking lot like old time. It explains why the PGS don't work as well as expected.

Aiming at the problem of parking guidance, we design parking guidance system based on Stackelberg game theory in this paper. The system is to build a game equilibrium between PGS and vehicles by modelling multi-round Stackelberg game, process data on web server and publish the recommendation on application UI. Finally, the performance of PGS is better and vehicles are more likely to accept the recommendation from system.

The rest of this paper is structured as follows. Section II reviews related work about study of PGS, Section III proposes the design of PGS. The evaluation and implement presented in Section IV. The conclusion is given in Section V.

\section{RELATED WORK}

The intelligence level of PGS has yet to be improved nowadays. There are some parking lots to realize the function of parking guidance by manual guidance; however, it brings the problems of low efficiency and high cost. Meanwhile, there are other parking lots help vehicles complete parking through the VMS, which vehicles can only obtain limit information. A few applications on the market provide parking guidance by pushing the information of the surrounding parking lots near destination, including Baidu map and Amap. Nevertheless, this way seems poor for the user experience comparing to recommend target parking lot directly. It is also inconvenient for vehicles to select target parking lot, since this way don't provider the number of available parking space in a specify parking lot.

In terms of theoretical research of parking guidance system, Sonqphon and Ronachai proposes a parking space monitoring management framework, with the function of idle

* This work was supported by Shenzhen S\&T Funding with Grant No. JCYJ20140417113430591, GJHZ20140417113430584, CXZZ2014 0527172356968, JSGG20150511104613104, JCYJ20160510154531467, and Guangdong S\&T Funding 2013B050800003, $2015 B 010106004$. 
parking prediction[2]; Ahmed Yousef and Ganesh Kumar introduced V2G intelligent scheduling technology into parking lot, studied the PSO optimal path algorithm and improved the efficiency of parking guidance[3], Lei Peng proposed a cost-effective parking space searching method by predicting with time-varying analysis of historical data[4].

\section{DESIGN}

We divide the parking guidance system into two parts according to the characteristic of UML. Structure diagram as one part represents the static architecture of system. Behaviour diagram as another part shows the dynamic process of the system; Stackelberg game model embody in this process.

\section{A. Static architecture}

The static architecture of this parking guidance system mainly contains two parts; they are front-end android application that is to present UI to user of vehicles and web server processing data by spider. Several functions have been implemented on application, including register and login of user, billing and payment, pushing information of alternative parking lots, recommending target parking lot and navigating etc. The pushing information contain the number of vacant parking spaces, location of parking lot etc. The processing data on web server is mainly obtained by spider, the function of spider contains city scanning, zone scanning and matching process. Finally, system obtain the overall basic data of parking lots in the specify city, the collection of alternative parking lots, and target parking lot of vehicles need to park. Figure1-2 show the class diagrams of application and spider respectively.

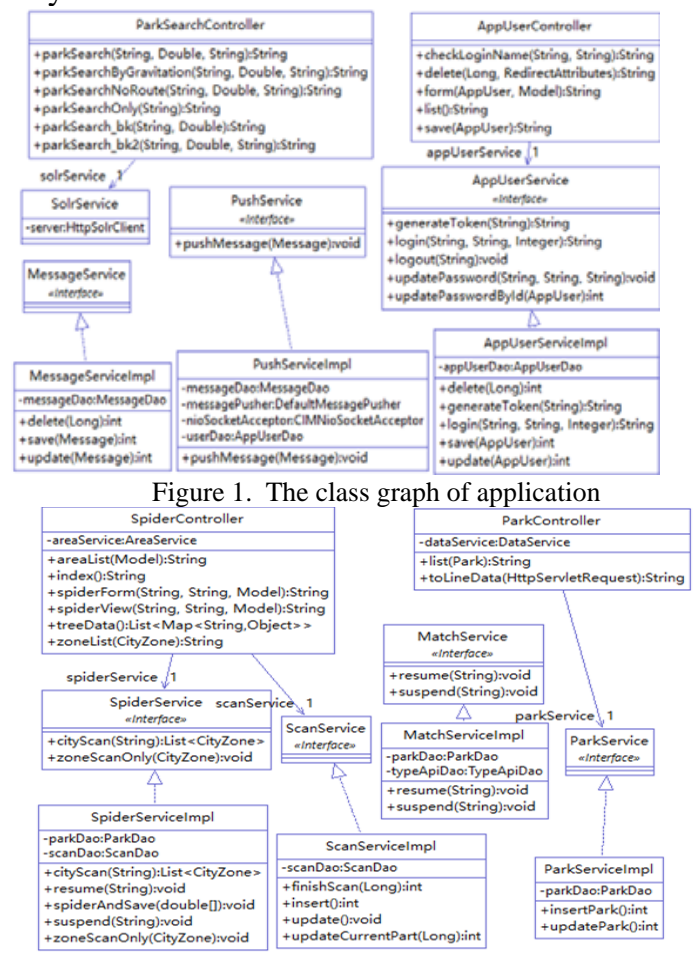

Figure 2. The class diagram of spider

\section{B. Dynamic procedure}

The relationship between the selection of vehicles and recommendation from PGS conform to the characteristics of the Stackelberg game. It is a dynamic process from seeding request by vehicles to recommending alternative parking lots and target parking lot from PGS and then to selecting target one, this dynamic process contains multiround game. The specify activity diagram is shown in Figure 3.

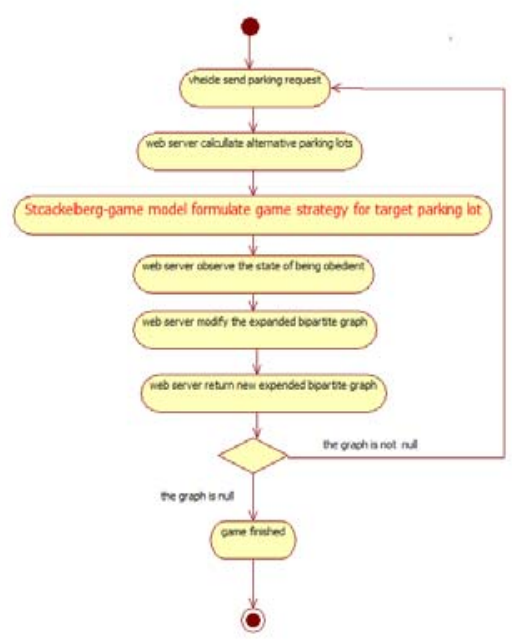

Figure 3 . The activity diagram of dynamic process

As shown in the above diagram, we propose the Stackelberg game model based on the relationship between vehicles and PGS, try to find out the equilibrium of parking guidance and recommend target parking lot to vehicles need to park, make sure that the system can obtain higher revenue and the vehicles are more likely to obey the recommendation from system.

As the leader of game, PGS publishes guidance information based on the principle of system optimal (SO). Maximizing the total parking revenue by using the method of network maximum flow. PGS considers the selection of vehicles into new publishing strategy to improve the performance of entire model. Vehicles, as a follower, make decision for selecting target parking lot based on the principle of Vehicles Equilibrium (UE), which it try to maximizing the parking revenue of individual vehicles. In this paper, we construct the evaluation function introduced by [4], thinking of revenue as satisfaction, to quantify the revenue between the vehicles $V_{\mathrm{j}}$ and parking lot $P_{i}$

$$
F\left(V_{\mathrm{j}}, P_{i}\right)=\alpha \ln \left(\frac{d_{p}}{\left|\sum_{i=1} E_{j}\right|} d_{i}\right)^{-1}+\beta \ln \left(\frac{E_{P}}{C_{P}}\right)^{-1}
$$

Where $d_{p}$ represents the distance between the two, $\mathrm{E}_{\mathrm{p}}$ is the amount of vacant parking space in $P_{i}, C_{P}$ represent the total number of vacancy in $P_{i}$. $\sum \mathrm{d}_{\mathrm{i}}$ represents the sum of distance between vehicles $V_{\mathrm{j}}$ and all alternative parking lots, The 
alternative parking lots in this paper is expressed as all optional parking lots within a radius range, it is a tolerable value for traveller. The effects of distance and the number of available parking spaces can be adjusted by modifying $\alpha$ and $\beta(0<\alpha<1,0<\beta<1)$.

In the Stackelberg game mode, vehicles have two choices, including obeying and disobeying recommendation from PGS. The PGS has only one choice that is to publish strategy. Therefore, we can conclude the matrix with revenue between PGS and vehicles based on the above theory. It is assumed that each vehicle will acquire revenue after one round of the game. The data about revenue is listed in the table I.

\begin{tabular}{|c|c|c|}
\hline vehicles & obey & disobey \\
\hline Strategy & $\sum_{j=1}^{m} F\left(V_{j}, P_{i}\right)$, & $\sum_{j=1}^{m} F\left(V_{j}, P_{k}\right)$, \\
& $F\left(v_{j}, p_{\mathrm{i}}\right)$ & $F\left(v_{j}, p_{\mathrm{k}}\right)$ \\
\hline
\end{tabular}

Where $F\left(V_{j}, P_{i}\right)$ represents the revenue that vehicles obeys the recommendation, $P_{i}$ represents the target parking lot recommended by the system, $F\left(V_{j}, P_{\mathrm{k}}\right)$ represents the revenue that vehicles disobeys the recommendation, $P_{\mathrm{k}}$ represents a parking lot that vehicles choose to disobey the recommendation.

The process of Stackelberg-game will last multi-round. Expanded bipartite graph was used to describe the process. At the beginning of the game, system publish the guidance strategy based on SO strategy, and then, count the obedience rate and remove some nodes that represent vehicles had obeyed the recommendation and adjacent sides. Finally, the system obtains a new expanded bipartite graph model. After the round of the game, system publish a new strategy to vehicles based on the new graph model, and then, vehicles make choice. After finished it, system updates nodes information and obtains a new expanded bipartite model again. Figure 4 show the changing process in one round of Stackelberg-game.
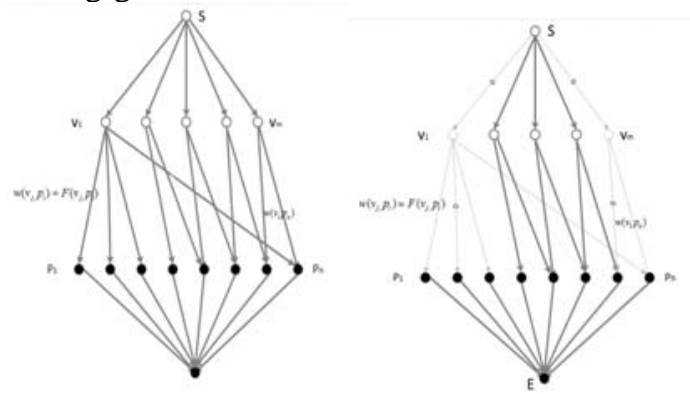

Figure 4. The changing process in one round of Stackelberg-game

This process of game is repeated. When the guidance strategy of PGS is consistent with the selections of vehicles, the game process reaches equilibrium.

\section{IMPLEMENT AND EVALUATION}

\section{A. Implement}

We design the application of parking guidance based on the third part theory, the specify UI show as Figure 5.

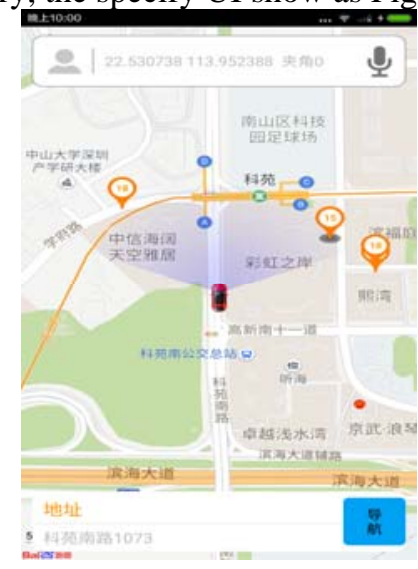

Figure 5. The UI of application

In Figure 5, the parking lot icon with shadow represent the recommended target parking lot. The number presented on icon is the estimated number of available parking spaces by proposed algorithm in reference [4].

We select Shenzhen city as scanning zone by spider. The spider crawls the basic information of parking lots, and inject those data into the model to predict the number of available parking spaces in one parking lot at a specify time. Figure 6 shows the scene of this process.

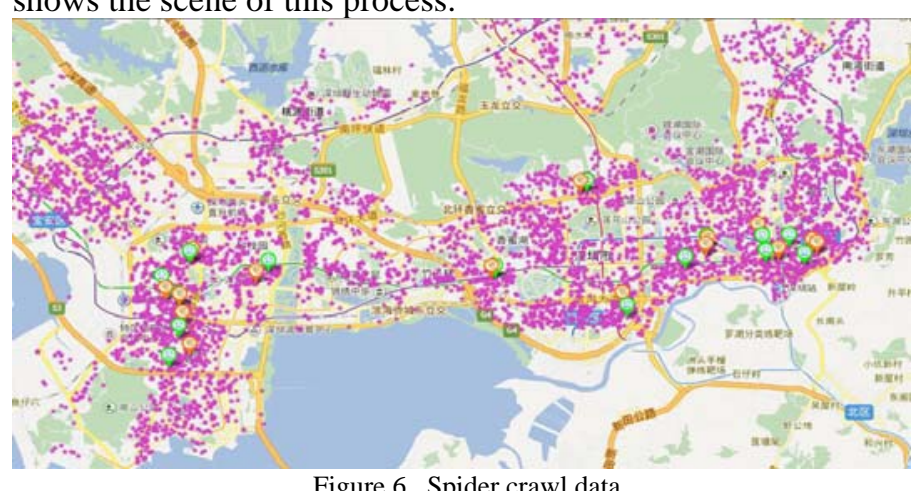

Figure 6. Spider crawl data

\section{B. Evaluation}

To verify the correctness and validity of the designed system, this paper establishes three mathematical models for three different traffic strategies. Experiments were proceeded in three different models respectively. Firstly, we do the experiment in the model using UE strategy. Secondly, we do the experiment in the model using SO strategy. Finally, the model using Stackelberg game strategy is to be chose. We also quote PO to represent the ratio of available parking space in whole parking spaces. After finishing those experiments, we analysed and compared the experimental results.

Experimental results are shown in 7-11. Firstly, Figure 7 shows relationship between average time of parking and proportion of used parking space corresponding to three 
different strategies. Secondly, Figure 8-10 show utilization of parking spaces at four moments. Figure 11 shows the changing process of standard deviation over time, it reflects well the fluctuation of utilization. In this experiment, we selected four parking lots, which is in the surrounding area of a science park, chose four moments of a working day as show on the horizontal axis in Figure 8. Those moments reflect well the process of traffic from smooth to congestion. $17: 00$ represents that $\mathrm{PO}$ is about 20\%, 17:30 represents that PO is about $50 \%, 18: 00$ represents that PO is near 70\%, 18:30 represents that $\mathrm{PO}$ is near $85 \%$.

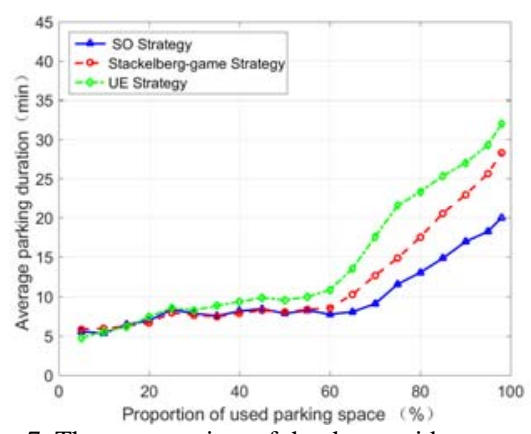

Figure 7 . The average time of the three guidance strategies
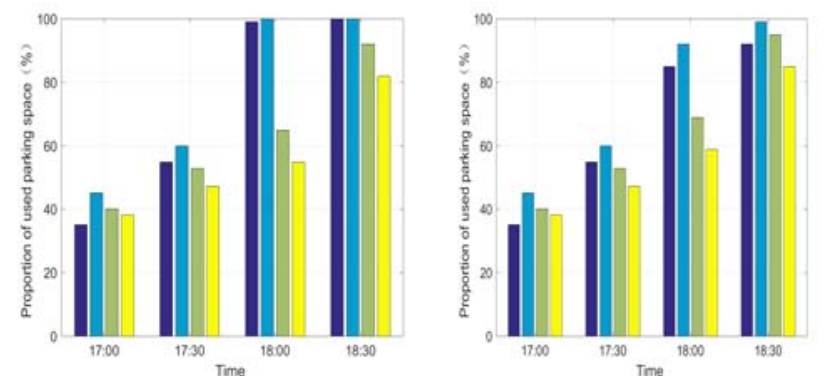

Figure 8. Vehicles equilibrium strategy

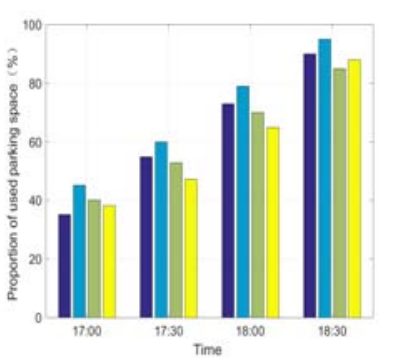

Figure10. System optimum strategy

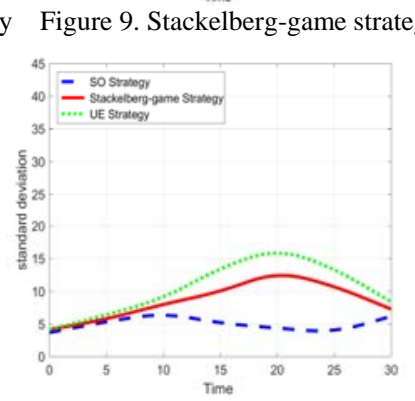

Figure 11. Standard deviation

In the initial conditions, the traffic is smooth and the number of the available parking space are adequate. The measured average time of parking is shorter, steady and tend to be consistent in three models as shown in Fig 7, because System using three strategies calculates matching relationship based on the same principle of the nearest distance. However, with the increasing of $\mathrm{PO}$, the number of available parking space is gradually scarce. The average time of parking in SO model is least. In contrast, biggest in UE model, intermediate using Stackelberg-game strategy. Since, the game model not only overcomes the problem of lacking fairness from SO strategy, but also improves the overall performance than UE strategy.
Figure 8-10 show that growth trend of utilization for parking lot are all steadily when the PO is less than 50\%. The reason is the same as the explanation about consistency in figure 7 . However, when the PO is greater than $50 \%$. Figure 8 shows that the growth trend fluctuates greatly, the main reason is that the growth trend is influenced by the location of parking lot. The better the location of parking lot, the faster the parking space in this parking lot are occupied. Figure 10 shows that the growth trend is also steadily. In that SO strategy calculates matching relationship based on the overall performance. Figure 9 shows that the trend is fluctuant, but it is not obvious by using standard deviation instead of fluctuation ratio for utilization.

\section{CONCLUSION}

After analysing disadvantage of existing parking guidance system, we propose a PGS based on Stackelberg game. This system solves two main problems; One is that SO strategy is lacking fairness and hard to implement. Another is that the efficiency of the UE strategy is low at rush hours. The evaluation is to verify the correctness and validity of the $d$ system. The results show that the system based on Stackelberg game can effectively reduce the average time of parking, achieves equilibrium of utilization for parking lot in region. However, this system considers only the game relationship between guidance system and vehicle. It ignores the game relationship between one vehicle and the other vehicle when the two vehicles compete for the same parking lot at the same time. Therefore, the next work will introduce the game between vehicles into this constructed PGS.

\section{REFERENCES}

[1] S. Pullola, P. K. Atrey, and A. E. Saddik, "Towards an Intelligent GPSBased Vehicles Navigation System for Finding Street Parking Lots," in 2007 IEEE International Conference on Signal Processing and Communications, 2007, pp. 1251-1254.

[2] J. Yue, T. Cheng, and M. Tai, "Demand Forecasting of Parking Lot Based on Discrete Choice Model in Planned Special Events," in 2009 International Conference on Management and Service Science, 2009, pp. 1-4.

[3] J. Chinrungrueng, S. Dumnin, and R. Pongthornseri, "iParking: A parking management framework," in 2011 11th International Conference on ITS Telecommunications, 2011, pp. 63-68.

[4] P. Lei and L. Huiyun, "Searching parking spaces in urban environments based on non-stationary Poisson process analysis," in 2016 IEEE 19th International Conference on Intelligent Transportation Systems (ITSC), 2016, pp. 1951-1956.

[5] W.Y. Szeto, Cooperative game approaches to measuring network reliability considering paradoxes. Transportation Research Part19,2011, pp. 229241.

[6] W. Li, X. Chen, T. Gu, and B. Zhou, "Study on differential dynamic Stackelberg game model and calculating method by GA for traffic guidance strategy," in 2009 Chinese Control and Decision Conference, 2009, pp. 2073-2078.

[7] L. Na and W. Chun, " A Stackelberg Game and Improved Logit Model based traffic guidance model," in Proceedings 2013 International Conference on Mechatronic Sciences, Electric Engineering and Computer (MEC), 2013, pp. 2382-2386.

[8] H. S. Mahmassani and R. Jayakrishnan, "System performance and vehicles response under real-time information in a congested traffic corridor," Transportation Research Part A: General, vol. 25, pp. 293-307, 1991/09/01 19 